\title{
Charmed states and flavour symmetry breaking
}

\author{
R. Horsley ${ }^{1, \star}$, Z. Koumi ${ }^{2}$, Y. Nakamura ${ }^{3}$, H. Perlt ${ }^{4}$, P. E. L. Rakow ${ }^{5}$, G. Schierholz ${ }^{6}$, A. Schiller ${ }^{4}$, \\ H. Stüben ${ }^{7}$, R. D. Young ${ }^{2}$, and J. M. Zanotti ${ }^{2}$ \\ for the QCDSF-UKQCD Collaborations \\ ${ }^{1}$ School of Physics and Astronomy, University of Edinburgh, Edinburgh EH9 3FD, UK \\ ${ }^{2}$ CSSM, Department of Physics, University of Adelaide, Adelaide SA 5005, Australia \\ ${ }^{3}$ RIKEN Advanced Institute for Computational Science, Kobe, Hyogo 650-0047, Japan \\ ${ }^{4}$ Institut für Theoretische Physik, Universität Leipzig, 04109 Leipzig, Germany \\ ${ }^{5}$ Theoretical Physics Division, Department of Mathematical Sciences, University of Liverpool, Liverpool L69 \\ $3 B X, U K$ \\ ${ }^{6}$ Deutsches Elektronen-Synchrotron DESY, 22603 Hamburg, Germany \\ ${ }^{7}$ Regionales Rechenzentrum, Universität Hamburg, 20146 Hamburg, Germany
}

\begin{abstract}
Extending the SU(3) flavour symmetry breaking expansion from up, down and strange sea quark masses to partially quenched valence quark masses allows an extrapolation to the charm quark mass. This approach leads to a determination of charmed quark hadron masses and decay constants. We describe our recent progress and give preliminary results in particular with regard to the recently discovered doubly charmed baryon (the $\Xi_{c c}^{++}$) by the LHCb Collaboration.
\end{abstract}

\section{Introduction}

Open charm baryon masses are presently the subject of much experimental and theoretical interest. In particular, most doubly charmed baryons (i.e. $c c q$ states) have not been experimentally observed even though as stable states in QCD they must exist. Indeed there has only been the observation of a candidate state, $\Xi_{c c}^{+}$(with quark content $c c d$ ) by the SELEX Collaboration, [1]. However this state was not seen by the BaBar, [2] or BELLE, [3]. Collaborations. Very recently, however, the LHCb Collaboration, [4], has announced a state - the $\Xi_{c c}^{++}$baryon - with a quark content $c c u$. It is unlikely that isospin breaking effects are significant (i.e. QED effects and $m_{u} \neq m_{d}$ ), or that the states have been missidentified so between the SELEX and LHCb result is an unexplained and puzzling mass difference of $\sim 100 \mathrm{MeV}$.

In this talk we shall describe the QCDSF-UKQCD approach to determining the hadron mass spectrum, with particular emphasis on the charm sector and the open doubly charmed baryon masses. This continues the programme initialised in [5].

$2+1$ flavour dynamical lattice simulations consist of two mass degenerate (i.e. $m_{u}=m_{d}$ ) light flavour $u, d$ quarks and a heavier flavour $s$ quark. Since the light quark masses are typically larger than the 'physical' masses required for the experimental spectrum, we are forced to consider how

^ Speaker, e-mail: rhorsley@ph.ed.ac.uk 
we can approach the physical $u, d, s$ quark masses. While many current simulations determine the physical $s$ quark mass and then extrapolate the $u, d$ quark masses to the physical quark mass, another possibility as suggested in [6] is to consider an $\mathrm{SU}(3)$ flavour breaking expansion from a point $m_{0}$ on the flavour symmetric line keeping the average quark mass $\bar{m}=\left(m_{u}+m_{d}+m_{s}\right) / 3$ constant. This procedure significantly reduces the number of expansion coefficients allowed. (Also, not considered here, the expansion coefficients remain the same whether we consider $m_{u} \neq m_{d}$ or $m_{u}=m_{d}$ and thus allows for the possibility of finding the pure QCD contribution to isospin breaking effects using just $n_{f}=2+1$ numerical simulations.)

As the charm quark is considerably heavier than the up, down and strange quarks an SU(4) flavour breaking expansion is poorly convergent (in distinction to the $\mathrm{SU}(3)$ flavour breaking expansion). Another possibility is to make independent SU(3) flavour breaking expansions in each charm sector (but this not a very unified approach). We adopt an intermediate aproach here, first noting that as the charm quark mass is much heavier than the $u, d$ and $s$ quark masses, it contributes little to the dynamics of the sea of the hadron. Thus we can regard the charm quark as a 'Partially Quenched' or PQ quark. By this we mean that the quark masses making up the meson or baryon have not necessarily the same mass as the sea quarks. Also the SU(3) flavour breaking expansion can also be extended to valence quark masses. As the expansion coefficients are just functions of $\bar{m}$, provided this is kept constant then the coefficients are unchanged. We shall say the 'Unitary Limit' when the masses of the valence quarks coincide with the sea quarks. PQ determinations have the advantage of not being expensive compared to dynamical simulations of the quarks. This can also help in the determination of the expansion coefficients as a wider range of quark masses than just the unitary masses can be used.

Briefly the method employed here is to first determine the expansion coefficients of the pseudoscalar mesons and octet baryons. Extrapolating to the physical pseudoscalar masses determines the 'physical' quark masses, which are then employed in the baryon expansions to determine the (open) charm masses.

\section{$2 \mathrm{SU}(3)$ flavour breaking expansions}

We shall only consider here hadrons which lie on the outer ring of their associated multiplet and not the central hadrons. So no mixing or quark-line disconnected correlation functions are considered here. The SU(3) flavour symmetry breaking expansions are given in terms of

$$
\delta \mu_{q}=\mu_{q}-\bar{m}, \quad \bar{m}=\frac{1}{3}\left(2 m_{l}+m_{s}\right),
$$

where $\mu_{q}$ are the PQ masses for quark $q$. In the unitary limit we have $\mu_{q} \rightarrow m_{q}$, where $m_{q}$ is the unitary quark mass (i.e. equal to the sea quark mass). Here we have the obvious constraint $2 \delta m_{l}+\delta m_{s}=0$.

For the pseudoscalar mesons with valence quarks $q=a$ and $b$, their masses are given to NLO (or quadratic in $\delta \mu_{q}$ by

$$
M^{2}(a \bar{b})=M_{0 \pi}^{2}+\alpha\left(\delta \mu_{a}+\delta \mu_{b}\right)+\beta_{0} \frac{1}{6}\left(2 \delta m_{l}^{2}+\delta m_{s}^{2}\right)+\beta_{1}\left(\delta \mu_{a}^{2}+\delta \mu_{b}^{2}\right)+\beta_{2}\left(\delta \mu_{a}-\delta \mu_{b}\right)^{2}+\ldots,
$$

(cubic or NNLO terms are given in [7]). The expansion coefficients are functions of $\bar{m}$ only, so if we keep $\bar{m}$ fixed we have constrained fits between the pseudoscalar mesons.

Numerically it is advantageous to use scale invariant quantities. A useful additional quantity with this method is to use flavour singlet or blind quantities, only defined in the unitary limit. There are many possibilities, for example for pseudoscalar meson quantities a convenient one is

$$
X_{\pi}^{2}=\frac{1}{3}\left(2 M_{K}^{2}+M_{\pi}^{2}\right)=M_{0 \pi}^{2}+O\left(\delta m_{l}^{2}\right)
$$


Now forming dimensionless ratios, $\tilde{M}^{2}=M^{2} / X_{\pi}^{2}, \tilde{\alpha}=\alpha / M_{0 \pi}^{2}, \tilde{\beta}_{i}=\beta_{i} / M_{0 \pi}^{2}, \ldots$ leads to the modified expansion

$$
\tilde{M}^{2}(a \bar{b})=1+\tilde{\alpha}\left(\delta \mu_{a}+\delta \mu_{b}\right)-\left(\frac{2}{3} \tilde{\beta}_{1}+\tilde{\beta}_{2}\right)\left(2 \delta m_{l}^{2}+\delta m_{s}^{2}\right)+\tilde{\beta}_{1}\left(\delta \mu_{a}^{2}+\delta \mu_{b}^{2}\right)+\tilde{\beta}_{2}\left(\delta \mu_{a}-\delta \mu_{b}\right)^{2}+\ldots
$$

Similarly for the outer ring of the baryon octet, we have the expansion

$$
\begin{aligned}
\tilde{M}_{\Sigma}^{2}(a a b)= & 1+\tilde{A}_{1}\left(2 \delta \mu_{a}+\delta \mu_{b}\right)+\tilde{A}_{2}\left(\delta \mu_{b}-\delta \mu_{a}\right) \\
& -\left(\tilde{B}_{1}+\tilde{B}_{3}\right)\left(2 \delta m_{l}^{2}+\delta m_{s}^{2}\right)+\tilde{B}_{1}\left(2 \delta \mu_{a}^{2}+\delta \mu_{b}^{2}\right)+\tilde{B}_{2}\left(\delta \mu_{b}^{2}-\delta \mu_{a}^{2}\right)+\tilde{B}_{3}\left(\delta \mu_{b}-\delta \mu_{a}\right)^{2},
\end{aligned}
$$

where we have collectively denoted these baryons with a $\Sigma$ index. Similarly to the meson case, we have formed dimensionless quantities by normalising with the singlet quantity

$$
X_{N}^{2}=\frac{1}{3}\left(M_{N}^{2}+M_{\Sigma}^{2}+M_{\Xi}^{2}\right)=M_{0 N}^{2}+O\left(\delta m_{l}^{2}\right) .
$$

The expansion given here to NLO (quadratic in $\delta \mu_{q}$ ). The expansion to NNLO (at next order with is given, for example, in [8] Appendix C. NNLO is used in the analysis presented here. Provided $m_{u}=m_{d}$ then for the $\Lambda$ we have

$$
\begin{aligned}
\tilde{M}_{\Lambda}^{2}\left(a a^{\prime} b\right)= & 1+\tilde{A}_{1}\left(2 \delta \mu_{a}+\delta \mu_{b}\right)-\tilde{A}_{2}\left(\delta \mu_{b}-\delta \mu_{a}\right) \\
& -\left(\tilde{B}_{1}+\tilde{B}_{3}\right)\left(2 \delta m_{l}^{2}+\delta m_{s}^{2}\right)+\tilde{B}_{1}\left(2 \delta \mu_{a}^{2}+\delta \mu_{b}^{2}\right)-\tilde{B}_{2}\left(\delta \mu_{b}^{2}-\delta \mu_{a}^{2}\right)+\tilde{B}_{4}\left(\delta \mu_{b}-\delta \mu_{a}\right)^{2},
\end{aligned}
$$

(the NNLO terms are also given in [8]. We shall use a prime, such as $a^{\prime}$, to denote a distinct quark, but with the same mass as quark $a$. (It turns out that the fits are slightly better if the square of the mass is used rather than just the mass. The SU3 flavour breaking expansions are valid for any function of the mass.)

Finally note that for open charm masses investigating mass splittings can give information on SU(3) mass splittings, as to LO there is no influence from the charm quark mass. For example for the pseudoscalar mesons

$$
\tilde{M}(a \bar{c})-\tilde{M}(b \bar{c})=\frac{1}{2} \tilde{\alpha}\left(\delta \mu_{a}+\delta \mu_{b}\right)+\ldots,
$$

while for the octet baryons we have

$$
\begin{aligned}
\tilde{M}(a a c)-\tilde{M}(b b c) & \left.=\left(\tilde{A}_{1}-\frac{1}{2} \tilde{A}_{2}\right)\right)\left(\delta \mu_{a}-\delta \mu_{b}\right)+\ldots \\
\tilde{M}(c c a)-\tilde{M}(c c b) & =\frac{1}{2}\left(\tilde{A}_{1}+\tilde{A}_{2}\right)\left(\delta \mu_{a}-\delta \mu_{b}\right)+\ldots
\end{aligned}
$$

\section{Lattice}

We use a $O(a)$ non-perturbatively improved clover fermion action, together with a Symanzik tree level improved glue. The fermions are also mildly stout smeared. Further details may be found in [9]. Thus the quark mass is given by

$$
\mu_{q}=\frac{1}{2}\left(\frac{1}{\kappa_{q}}-\frac{1}{\kappa_{0 c}}\right),
$$

where $\kappa_{q}$ is the hopping parameter, $\kappa_{0}$ is the hopping parameter along the symmetric line with $\kappa_{0 c}$ being its chiral limit. Note that for $\delta \mu_{q}$, the 'distance' from the initial SU3 flavour symmetry point, $\kappa_{0 c}$, cancels and so does not have to be determined. We have presently analysed four lattice spacings where $a \sim 0.052-0.074 \mathrm{fm}$, but are aiming for five spacings.

In the LH panel of Fig. 1 we show $X_{S}^{\text {lat } 2}$ for various singlet quantities, $S=t_{0}, N, w_{0}, \rho, \pi$. This can be used to determine both the lattice spacing and $\kappa_{0}$ - the point on the $\mathrm{SU}(3)$ flavour symmetric, to 

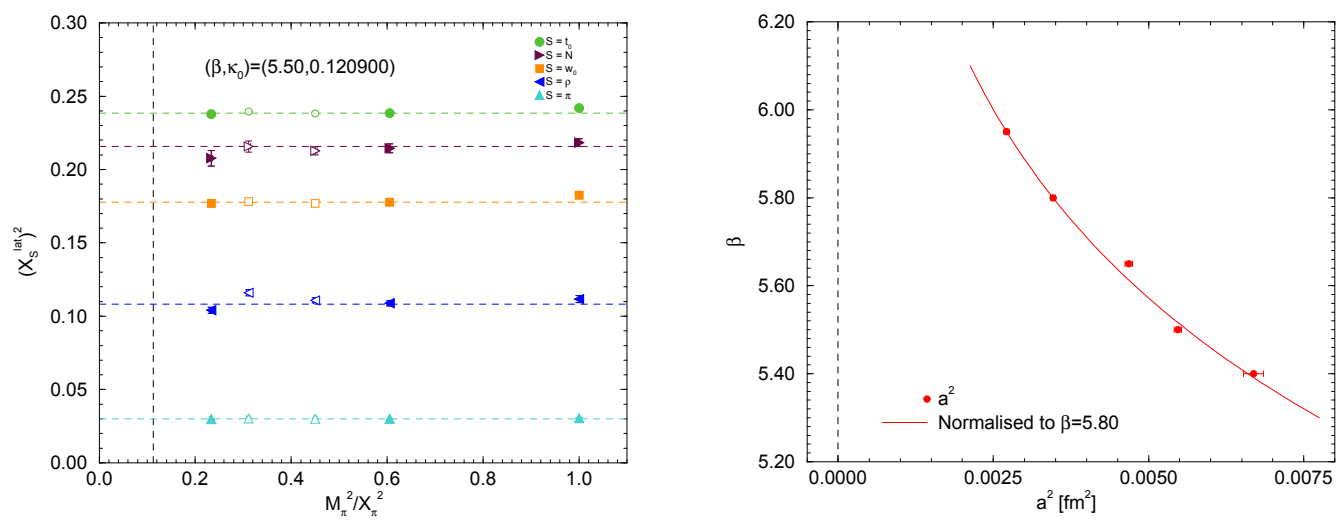

Figure 1. Left panel: $\left.X_{S}^{\text {lat }}\right)^{2}$ for $S=t_{0}, N, w_{0}, \rho$ and $\pi$ along the unitary line, from the symmetric point $\delta m_{l}=0$ down to the physical point (vertical dashed line) together with constant fits (for $\beta=5.50, \kappa_{0}=0.120900$ ). Right panel: $a^{2}(\beta)$ values normalised to $a^{2}(5.80)\left(a^{2} \sim 0.035 \mathrm{fm}^{2}\right)$. The line shows the two-loop $\beta$-function.

start the path to the physical point. [10]. For example, this could be achieved by tuning $X_{\pi}^{\text {lat } 2} / X_{N}^{\text {lat } 2}$ to its 'physical' value, using wherever possible the 'pure' QCD values in FLAG3, [11], otherwise from the PDG, [12]. In the RH panel we show $a^{2}$ values normalised by the value at $\beta=5.80$ using the two loop $\beta$-function.

We first determine the pseudoscalar meson expansion coefficients. In the LH panel of Fig. 2 we show $\tilde{M}(a \bar{b})=M^{2}(a \bar{b})^{2} / X_{\pi}^{2}$ versus $\left(\delta \mu_{a}+\delta_{b}\right) / 2\left(\right.$ for $\left.\left(\beta, \kappa_{0}\right)=(5.80,0.122810)\right)$. To illustrate the
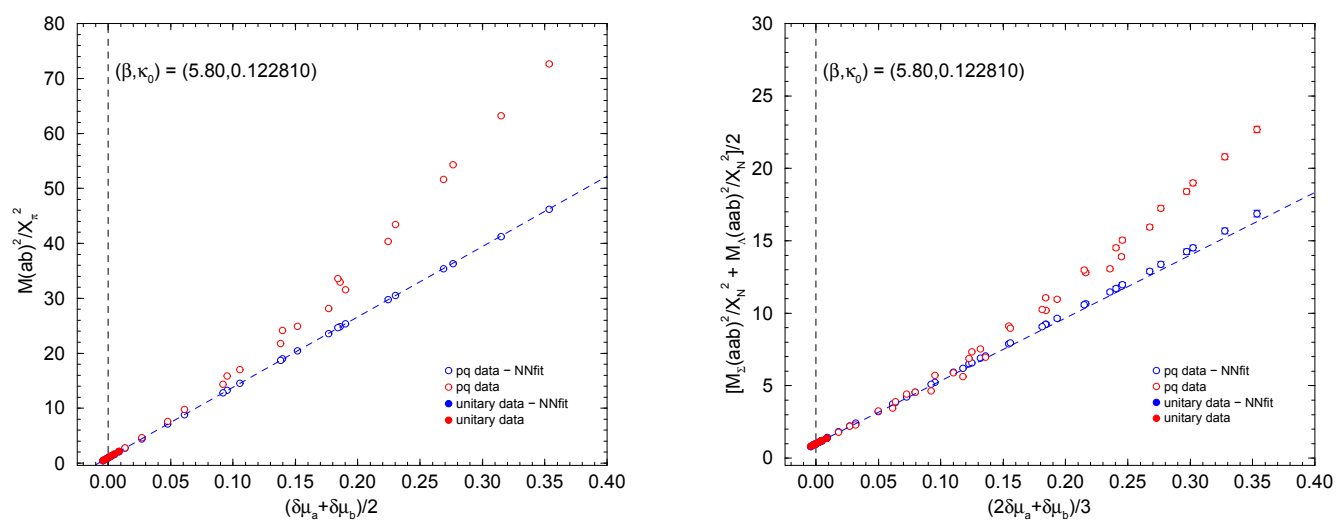

Figure 2. Left panel: $\tilde{M}(a \bar{b})=M^{2}(a \bar{b})^{2} / X_{\pi}^{2}$ versus $\left(\delta \mu_{a}+\delta \mu_{b}\right) / 2$ for $\left(\beta, \kappa_{0}\right)=(5.80,0.122810)$. The PQ masses are given by opaque red circles (the unitary data by filled red circles). Removing the NN term (leaving the LO terms) gives the blue opaque circles and linear fit term. Right panel: Similarly for $\left(\tilde{M}_{\Sigma}^{2}(a a b)+\tilde{M}_{\Lambda}^{2}\left(a a^{\prime} b\right)\right) / 2$ versus $\left(2 \delta \mu_{a}+\delta \mu_{b}\right) / 3$.

fit (which at LO is a function $\left(\delta \mu_{a}+\delta \mu_{b}\right) / 2$ only) after fitting we subtract the higher order terms to leave only the LO term. Similarly in the RH panel of Fig. 2 we show the equivalent result for the 
octet baryon case by plotting $\left(\tilde{M}_{\Sigma}^{2}(a a b)+\tilde{M}_{\Lambda}^{2}\left(a a^{\prime} b\right)\right) / 2$ versus $\left(2 \delta \mu_{a}+\delta \mu_{b}\right) / 3$. To determine $\delta m_{l}^{*}$ the 'physical' quark mass we set the pseudoscalar meson combination $M_{\pi}^{2} / X_{\pi}^{2}$ to its physical value. Similarly we use $M_{\eta_{c}}^{2} / X_{\pi}^{2}$ to determine $\delta m_{c}^{*}$.

Although we have scanned in detail the appropriate region to determine the initial point, $\kappa_{0}$ for the trajectory some fine tuning is possible. Bearing in mind that changes will come mostly from the valence quark mass (rather than the sea quark masses), we do not consider exactly the unitary limit, but allow $\delta \mu_{l}^{*}, \delta \mu_{s}^{*}$ to be slightly different to $\delta m_{l}^{*}, \delta m_{s}^{*}$ by fitting $X_{\pi}^{p q 2} / X_{N}^{p q 2}$ and $M_{\pi}^{p q 2} / X_{\pi}^{p q 2}$ to their physical values, while keeping the splitting between $\delta \mu_{l}^{*}, \delta \mu_{s}^{*}$ the same as $\delta m_{l}^{*}, \delta m_{s}^{*}$. Presently we see little difference, [13], and shall not discuss this further here.

\section{Results}

After having determined the expansion coefficients and the 'physical' quark masses, we can first determine the open charm pseudsoscalar mesons. All errors shown in the following plots are statistical (however we expect systematic errors, if any, to be small). In the LH panel of Fig. 3 we show preliminary results for the continuum extrapolation of the $D_{s}(s \bar{l}), D(c \bar{l})$ masses and their mass differ-
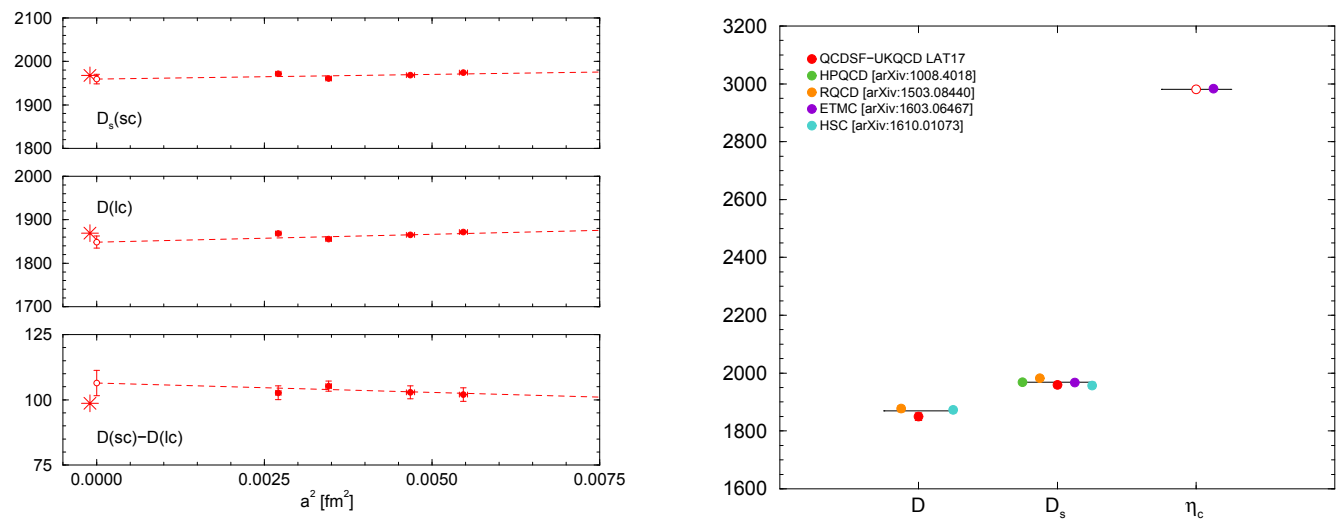

Figure 3. Left panel: Preliminary $D_{s}(s \bar{c})$, and $D(l \bar{c})$ masses (upper and middle plot) together with their mass difference (lower plot) versus $a^{2}$, together with linear fits in $a^{2}$. The experimental results are shown with a star (slightly displaced in the -ve- $x$ direction for clarity). Right panel: Comparison of the extrapolated results to other recent results, [14-17]. The opaque red circle for the $\eta_{c}$ mass is just to indicate that this has been used in the determination of the charm quark mass.

ence (upper to lower plots). Also shown is a linear extrapolation in $a^{2}$ to the continuum limit. The phenomenological values are denoted by a star. The mass differences in particular are sensitive to unknown $u-d$ quark mass difference and QED effects (the present computation is for pure QCD only). From the plots we see that there do not seem to be strong scaling violations present. The RH panel of Fig. 3 shows a comparison with some other determinations.

Presently we have determined charm baryon states with nucleon-like wavefunctions $\mathcal{B}=$ $\epsilon q\left(q^{T} C \gamma_{5} c\right)(q=l, s)$. In Table 1 we show the possible states in the isospin symmetric limit, $m_{u}=m_{d} \equiv m_{l}$. (A prime denotes a distinct quark in the wavefunction, but with the same mass.) Thus in the charm sector, we have presently investigated: $\Sigma_{c}(l l c), \Omega_{c}(s s c), \Xi_{c c}(c c l)$ and $\Omega_{c c}(c c s)$.

In the left panel of Fig. 4 we show the $C=1$ open charmed baryons $\Omega_{c}(s s c), \Sigma_{c}(l l c)$ masses, 


\begin{tabular}{ccccc}
$C$ & $S$ & $I$ & baryon & wavefunction \\
\hline 0 & 0 & $\frac{1}{2}$ & $N\left(l l l^{\prime}\right)$ & $\epsilon\left(l^{T} C \gamma_{5} l^{\prime}\right) l$ \\
0 & 1 & 1 & $\Sigma(l l s)$ & $\epsilon\left(l^{T} C \gamma_{5} s\right) l$ \\
0 & 2 & $\frac{1}{2}$ & $\Xi(s s l)$ & $\epsilon\left(s^{T} C \gamma_{5} l\right) s$ \\
0 & 1 & 0 & $\Lambda\left(l l^{\prime} s\right)$ & $\frac{1}{\sqrt{6}} \epsilon\left[2\left(l^{T} C \gamma_{5} l^{\prime}\right) s+\left(l^{T} C \gamma_{5} s\right) l^{\prime}-\left(l^{T} C \gamma_{5} s\right) l\right]$ \\
\hline 1 & 0 & 1 & $\Sigma_{c}(l l c)$ & $\epsilon\left(l^{T} C \gamma_{5} c\right) l$ \\
1 & 1 & $\frac{1}{2}$ & $\Xi_{c}^{\prime}(l s c)$ & $\frac{1}{\sqrt{2}} \epsilon\left[\left(s^{T} C \gamma_{5} c\right) l+\left(l^{T} C \gamma_{5} c\right) s\right]$ \\
1 & 2 & 0 & $\Omega_{c}(s s c)$ & $\epsilon\left(s^{T} C \gamma_{5} c\right) s$ \\
1 & 0 & 0 & $\Lambda_{c}\left(l l^{\prime} c\right)$ & $\frac{1}{\sqrt{6}} \epsilon\left[2\left(l^{T} C \gamma_{5} l^{\prime}\right) c+\left(l^{T} C \gamma_{5} c\right) l^{\prime}-\left(l^{T} C \gamma_{5} c\right) l\right]$ \\
1 & 1 & $\frac{1}{2}$ & $\Xi_{c}(c s l)$ & $\frac{1}{\sqrt{6}} \epsilon\left[2\left(s^{T} C \gamma_{5} l\right) c+\left(s^{T} C \gamma_{5} c\right) l-\left(l^{T} C \gamma_{5} c\right) s\right]$ \\
\hline 2 & 0 & $\frac{1}{2}$ & $\Xi_{c c}(c c l)$ & $\epsilon\left(c^{T} C \gamma_{5} l\right) c$ \\
2 & 1 & 0 & $\Omega_{c c}(c c s)$ & $\epsilon\left(c^{T} C \gamma_{5} s\right) c$
\end{tabular}

Table 1. The possible $C=0,1$ and 2 baryon octet states in the isospin symmetric limit, $m_{u}=m_{d} \equiv m_{l}$. A prime is used to denote a distinct quark, but with the same mass.
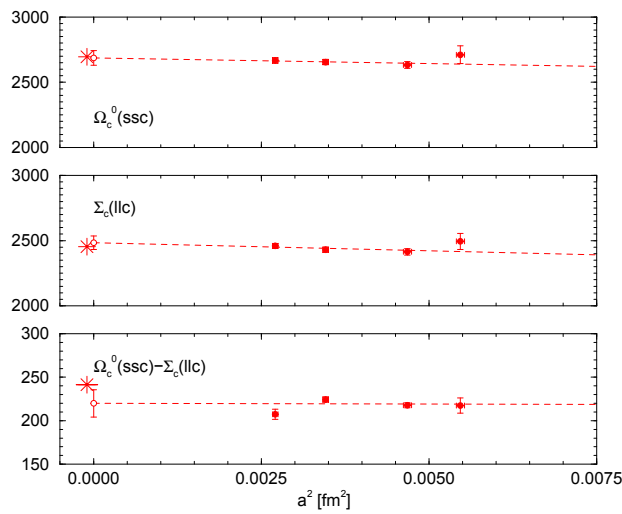
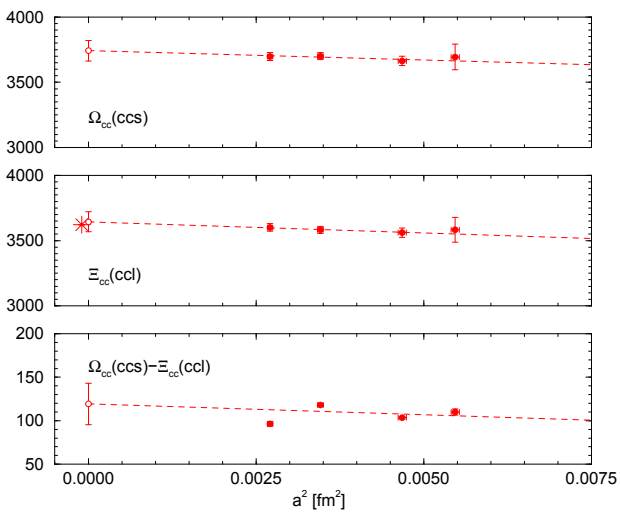

Figure 4. Left panel: Preliminary $\Omega_{c}(s s c), \Sigma_{c}(l l c)$ and mass difference results versus $a^{2}$ (upper to lower plots), together with linear extrapolations to the continuum limit. Right panel: similarly for the $C=2$ open charm states $\Sigma_{c}(l l c), \Omega_{c}(s s c)$. The LHCb result, [4], is also shown in the centre plot.

together with their mass splitting. For the single open charm states there is reasonable agreement with the experimental results. Again, as for the open charm pseudoscalar masses, the scaling violations seem to be moderate.

In the right panel of Fig. 4 we show the $C=2$ open charmed baryons. We examine the result for the mass of the $\Xi_{c c}$ in greater detail in Fig. 5. Our tentative conclusion is that our results can differentiate between LHCb and SELEX and tend to support the LHCb result. (As mentioned before we assume that isospin breaking results are small.) This also is in agreement with other recent results, such as [15, 18-20]. 


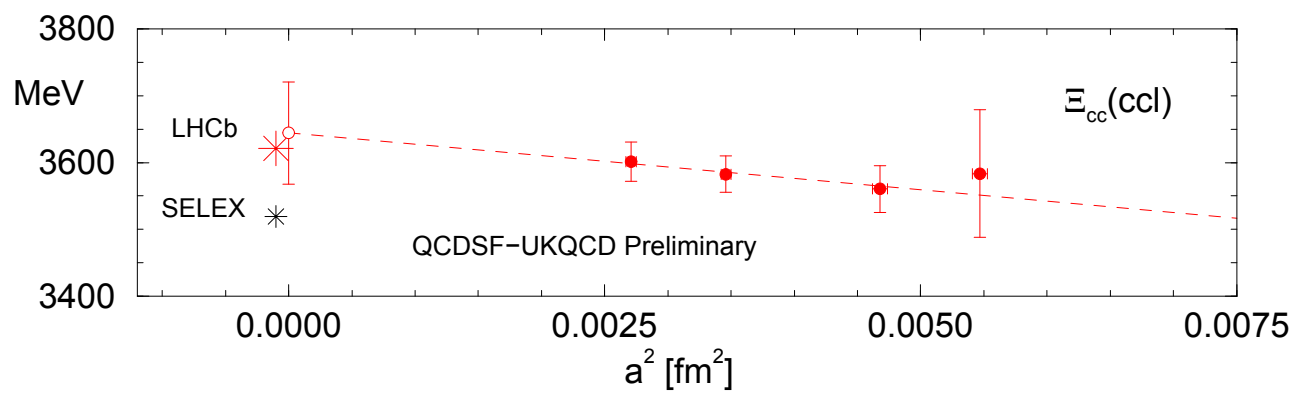

Figure 5. An enlarged plot for the $\Xi_{c c}$ mass from Fig. 4. Also indicated are the LHCb result, [4] and the SELEX result, [1].

\section{Conclusions}

For $u, d, s$ quarks, have developed a method to approach the physical point on a path starting from a point on the SU(3) flavour symmetric line. We have developed precise $S U(3)$ flavour symmetry breaking expansions - nothing is ad-hoc. The expansions have been extended - PQ or when mass valence quarks $\neq$ mass sea quarks). This enables a better determination of the expansion coefficients and also allows the expansion to be applied in the region of the $c$ quark mass. We have data for four lattice spacings and have applied the method to determine some open charm state masses, in particular the recently discovered $C=2$ open charm state $\Xi_{c c}^{++}$. The preliminary results are displayed in Fig. 5 .

In the future we plan to extend the results to the other states shown in Table 1 increasing the number of lattice spacing to five. Furthermore, the expansions also are valid for $m_{u} \neq m_{d}$, so with the determined coefficients it may be possible to investigate pure QCD isospin breaking effects, in particular to generalise to include mixing, [8], for example to $\Sigma_{c}^{+}-\Lambda_{c}^{+}, \Xi_{c}^{0}-\Xi_{c}^{\prime 0}$ mixing. Further possibilities include an investigation of the charmed baryon decuplet and QED effects. For the latter we are in the process of investigating using our recently generated ensemble of fully dynamical QCD+QED configurations $[21,22]$.

\section{Acknowledgements}

The numerical configuration generation (using the BQCD lattice QCD program [23]) and data analysis (using the Chroma software library [24]) was carried out on the IBM BlueGene/Qs using DIRAC 2 resources (EPCC, Edinburgh, UK), and at NIC (Jülich, Germany) and the SGI ICE 8200 and Cray XC30 at HLRN (The North-German Supercomputer Alliance) and on the NCI National Facility in Canberra, Australia (supported by the Australian Commonwealth Government). HP was supported by DFG Grant No. SCHI 422/10-1 and GS was supported by DFG Grant No. SCHI 179/8-1. PELR was supported in part by the STFC under contract ST/G00062X/1 and JMZ was supported by the Australian Research Council Grant No. FT100100005 and DP140103067. We thank all funding agencies. 


\section{References}

[1] M. Mattson et al., [SELEX Collaboration], Phys. Rev. Lett. 89 (2002) 112001, [arXiv:hepex/0208014]; A. Ocherashvili et al., [SELEX Collaboration], Phys. Lett. B 628 (2005) 18, [arXiv:hep-ex/0406033].

[2] B. Aubert et al., [BaBar Collaboration], Phys. Rev. D 74 (2006) 011103, [arXiv:hepex/0605075].

[3] R. Chistov et al., [BELLE Collaboration], Phys. Rev. Lett. 97 (2006) 162001, [arXiv:hepex/0606051]; Y. Kato et al., [BELLE Collaboration], Phys. Rev. D 89 (2014) 052003, [arXiv:1312.1026 [hep-ex]].

[4] R. Aaij et al., [LHCb Collaboration], Phys. Rev. Lett. 119 (2017) 112001, arXiv:1707.01621 [hep-ex].

[5] R. Horsley et al., [QCDSF-UKQCD Collaborations], PoS (LATTICE 2013) (2014) 249, arXiv:1311.5010 [hep-lat].

[6] W. Bietenholz et al., [QCDSF-UKQCD Collaborations], Phys. Rev. D 84 (2011) 054509, [arXiv:1102.5300 [hep-lat]]; W. Bietenholz et al., [QCDSF-UKQCD Collaborations], Phys. Lett. B 690 (2010) 436, [arXiv:1003.1114 [hep-lat]].

[7] R. Horsley et al., [QCDSF-UKQCD Collaboration], Phys. Rev. D 86 (2012) 114511, [arXiv:1206.3156 [hep-lat]].

[8] R. Horsley et al., Phys. Rev. D 91 (2015) 074512, [arXiv:1411.7665 [hep-lat]].

[9] N. Cundy et al., [QCDSF-UKQCD Collaboration], Phys. Rev. D 79 (2009) 094507, [arXiv:0901.3302[hep-lat]].

[10] V. G. Bornyakov et al., [QCDSF-UKQCD Collaborations], arXiv:1508.05916[hep-lat].

[11] S. Aoki et al., [FLAG3], Eur. Phys. J. C 77 (2017) 112, [arXiv:1607.00299 [hep-lat]].

[12] C. Patrignani et al. (Particle Data Group), Chin. Phys. C 40 (2016) 100001 and 2017 update.

[13] QCDSF-UKQCD Collaborations, in preparation.

[14] C. T. H. Davies et al., Phys. Rev. D 82 (2010) 114504, [arXiv:1008.4018 [hep-lat]].

[15] P. Pérez-Rubio et al., Phys. Rev. D 92 (2015) 034504, [arXiv:1503.08440 [hep-lat]].

[16] K. Cichy et al., Phys. Rev. D 94 (2016) 094503, [arXiv:1603.06467 [hep-lat]].

[17] G. K. C. Cheung et al., [Hadron Spectrum Collaboration], JHEP 1612 (2016) 089, [arXiv:1610.01073 [hep-lat]].

[18] Y. Namekawa et al., [PACS-CS Collaboration], Phys. Rev. D 87 (2013) 094512, [arXiv:1301.4743 [hep-lat]].

[19] C. Alexandrou et al., Phys. Rev. D 90 (2014) 074501, [arXiv:1406.4310 [hep-lat]].

[20] C. Alexandrou et al., Phys. Rev. D 96 (2017) 034511, [arXiv:1704.02647 [hep-lat]].

[21] R. Horsley et al., J. Phys. G 43 (2016) 10LT02, [arXiv:1508.06401 [hep-lat]].

[22] R. Horsley et al., JHEP 1604 (2016) 093, [arXiv:1509.00799 [hep-lat]].

[23] Y. Nakamura and H. Stüben, Proc. Sci. Lattice 2010 (2010) 040, arXiv:1011.0199 [hep-lat]; poster at Lattice 2017.

[24] R. G. Edwards and B. Joó, Nucl. Phys. Proc. Suppl. 140 (2005) 832, arXiv:hep-lat/0409003. 